\title{
Coherent imaging of a pure phase object with classical incoherent light
}

\author{
M. Bache, ${ }^{*}$ D. Magatti, F. Ferri, A. Gatti, E. Brambilla, and L. A. Lugiato \\ CNR-INFM-CNISM, Dipartimento di Fisica e Matematica, Università dell'Insubria, Via Valleggio 11, 22100 Como, Italy
}

(Received 21 December 2005; published 3 May 2006)

\begin{abstract}
By using the ghost imaging technique, we experimentally demonstrate the reconstruction of the diffraction pattern of a pure phase object by using the classical correlation of incoherent thermal light split on a beam splitter. The results once again underline that entanglement is not a necessary feature of ghost imaging. The light we use is spatially highly incoherent with respect to the object $(\approx 2 \mu \mathrm{m}$ speckle size $)$ and is produced by a pseudo-thermal source relying on the principle of near-field scattering. We show that in these conditions no information on the phase object can be retrieved by only measuring the light that passed through it, neither in a direct measurement nor in a Hanbury Brown-Twiss (HBT) scheme. In general, we show a remarkable complementarity between ghost imaging and the HBT scheme when dealing with a phase object.
\end{abstract}

DOI: 10.1103/PhysRevA.73.053802

PACS number(s): 42.50.Dv, 42.50.Ar, 42.30.Va

\section{INTRODUCTION}

Ghost imaging is a technique which allows one to perform coherent imaging with incoherent light by exploiting the spatial correlation between two beams created by, e.g., parametric down conversion (PDC) [1-20]. Each of the correlated beams is sent through a distinct imaging system, traditionally called the test and the reference arm. In the test arm an object is placed and information about the object is recreated from the spatial correlation function between the test and reference arm.

One of the most striking features of the ghost imaging technique is that since the two beams are spatially incoherent no phase-sensitive information about an object can be extracted by observing a single beam only. This means that the diffraction pattern of an object that substantially alters the phase of the incoming light cannot be observed in any way in the test arm. Nonetheless, because of the mutual spatial correlation between the two beams, we will show that the diffraction pattern may be reconstructed through the spatial correlation between the two beams. In other words, despite the beams being incoherent, the coherence between them makes the imaging coherent: the scheme is therefore capable of doing coherent imaging with incoherent light.

On the other hand, the diffraction pattern of an object that only alters the amplitude of the light, such as a Young's double slit, can be extracted from the intensity distribution of the object arm only, even when the object is illuminated with a spatially incoherent beam. One may do so by measuring the autocorrelation of the transmitted field as observed in the far zone of the object. This can be conveniently done by using the Hanbury Brown-Twiss (HBT) scheme [21]. Thus, the ghost imaging technique is not required in this case, and one may stick to using a single beam only.

It is therefore interesting to look beyond the case of an amplitude-only object. As an extreme case, in this work we

\footnotetext{
*Present address: COM·DTU, Technical University of Denmark, DK-2800 Lyngby, Denmark.
}

want to observe the diffraction pattern of a pure phase $o b$ ject, an object that only alters phase information $[9,13,15]$.

Initially the possibility of performing ghost imaging was ascribed to the presence of spatial entanglement between the two arms $[3-5,8]$. Lately this view has been challenged by many groups $[10,12,13,17-20,22-28]$ (to cite but a few). Our group in particular has produced numerous publications, showing both theoretically [13] and experimentally [23-25] that basically all features offered by entangled ghost imaging can be mimicked by using a proper scheme that exploits classically correlated beams: the correlation between the beams is in this scheme created by dividing an incoherent pseudo-thermal speckle beam on a beam splitter (BS). The two outgoing beams are then still incoherent on their own but, since they are (classical) copies of each other, they have a high mutual spatial coherence. We showed that the only feature that cannot be mimicked by classical correlation is the $100 \%$ visibility of the information, which can be in principle achieved only with entangled photons; however, in the classical case the visibility is still good enough to effectively reconstruct the information [13,17-20,23-25]. An important outcome of our analyses is that the entangled ghost imaging and our classical ghost imaging have the common feature of providing coherent imaging using incoherent light [13]. Thus, both schemes should be able to reconstruct the diffraction pattern of any object, altering amplitude as well as phase [13]. We experimentally confirmed this prediction in the case of an amplitude object [23].

An experiment of Abouraddy et al. [9] demonstrated the reconstruction of the ghost diffraction pattern of a pure phase object using the entangled photon pairs produced by spontaneous parametric down conversion, which represents an optical field that lacks second-order spatial coherence but is endowed with higher order spatial coherence. The introduction of Ref. [9] claims that our ghost diffraction experiment with classical thermal light reported in Ref. [23] could be performed only because effectively the thermal light was endowed with second-order spatial coherence. This might suggest that coherent imaging with spatially incoherent light is not possible with split thermal light. This interpretation of our experiment [23] was not correct, because the light we 
used there was indeed spatially incoherent to a high degree. The viewpoint of Ref. [9] was challenged in Refs. [24,25], where not only did we experimentally demonstrated again the ghost diffraction of an amplitude object with classical incoherent light, but also showed that the spatial incoherence of the light is a necessary ingredient to carry out the task. In this work we will finally demonstrate experimentally and theoretically that the claim of Ref. [9] is not correct also in the case of a pure phase object.

We will show that the diffraction pattern of a commonly used pure phase object, a transmission grating beam splitter (TGBS), can be reconstructed from the classical correlations between two highly spatially incoherent beams generated by splitting a speckle beam on a BS. In order to render the light impinging on the object incoherent with respect to the object, we have to produce speckles of size on the order of $2.0 \mu \mathrm{m}$, and we achieve it through the so-called near-field scattering technique $[29,30]$. Incidentally we remark that such a small speckle size, which implies a spatial resolution on the order of $2.0 \mu \mathrm{m}$ (see, e.g., Ref. [23]), has no precedent to our knowledge in ghost imaging schemes, either with thermal or entangled beams.

With such a degree of spatial incoherence, we will verify that no information about the phase object diffraction pattern is present in the test arm, neither in the far zone intensity distribution nor in its autocorrelation, which is equivalent to a HBT type of measurement. The information gradually appears in the test arm as the degree of spatial coherence increases. The converse holds for the ghost diffraction scheme: the information on the phase object can be retrieved from the correlation between the test and the reference arm only when the light is spatially incoherent, and it disappears when increasing the coherence. From these results we will conclude that the claim of Ref. [9] is incorrect for the ghost imaging scheme, which indeed works as a coherent imaging scheme only because of incoherence. This claim could be possibly applied to the HBT scheme, which in the case of a phase object works as a coherent imaging system only when the light is coherent.

Thus, there exist remarkable differences between the ghost imaging and the HBT schemes, which will be clarified in the present work.

The paper is organized as follows. Section II describes the experimental setup. Section III is devoted to theoretical results, with Sec. III A giving the basics of the theory of the ghost diffraction, Sec. III B discussing the properties of the chosen object, and Sec. III C presenting some numerical results used as a guideline for the experimental implementation. In fact, the speckles needed are so small that we have to use a near-field scattering technique to produce them. This technique, along with its experimental implementation, is described in Sec. IV A. Section IV B finally presents the experimental results of ghost diffraction and of the HBT scheme with incoherent illumination, while Sec. IV C present results illustrating the transition from incoherent to coherent illumination. Section V concludes the work.

\section{THE SETUP}

The ghost diffraction setup used in our experiment is schematically shown in Fig. 1. A pseudo-thermal source gen-

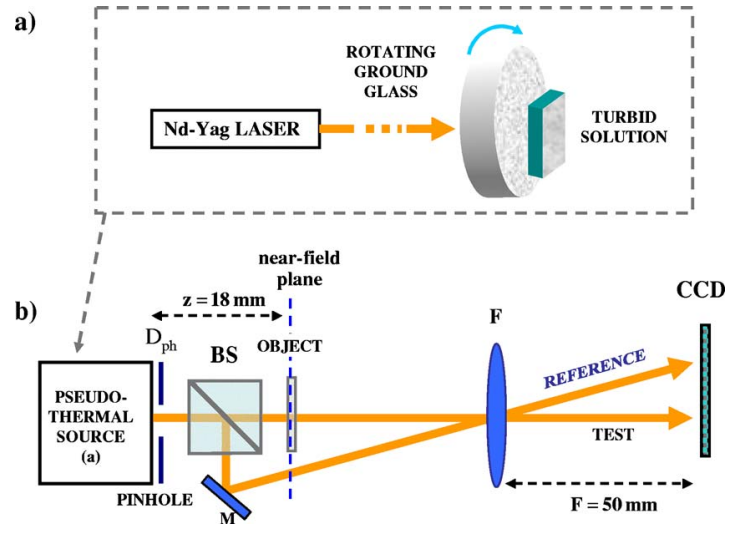

FIG. 1. (Color online) Experimental setup for ghost diffraction of a pure phase object using classical pseudo-thermal light. See text for details.

erates a chaotic speckle beam, which enters a balanced $50 / 50 \mathrm{BS}$ (the side of the glass cube is $12.5 \mathrm{~mm}$ ). The pseudo-thermal source [Fig. 1(a)] consists of a large collimated laser beam (frequency doubled Nd:YAG laser, $\lambda=0.532 \mu \mathrm{m}$, diameter $D \approx 10 \mathrm{~mm}$ ) illuminating a slowly rotating ground glass followed by a square cell containing a colloidal turbid solution. The transversal size of the source is delimited by a pinhole of diameter $D_{\mathrm{ph}}=4.5 \mathrm{~mm}$, which is placed at the exit face of the turbid cell. A detailed description of the thermal source together with the features of the speckles that it generates will be given in Sec. IV A.

The BS divides the pseudo-thermal radiation into two "twin" speckle beams: the transmitted one is used for the test arm and is sent onto the object located right after the BS at a distance $z=18 \mathrm{~mm}$ from the source. The reflected beam is used for the reference arm; the mirror $M$ deflects it towards the detector in a direction forming a small angle with the test arm. Note that, thanks to the double reflection of the reference arm, the test and reference beams on the detector are not mutually reversed, but are each other's replica.

Both beams are collected by the central part of the lens $F$ (focal length $F=50 \mathrm{~mm}$ ) and their intensity distributions are detected by a charged coupled device (CCD) sensor placed in the focal plane of the lens. Due to the small angle between the two arms, the corresponding light spots fall onto different non-overlapping regions of the CCD sensor.

The data are acquired with an exposure time $\approx 0.1 \mathrm{~ms}$, much shorter than the speckle coherence time $\tau_{\text {coh }} \approx 10 \mathrm{~ms}$ (see Sec. IV A), allowing the recording of high-contrast speckle patterns. The frames are grabbed at a rate of $5 \mathrm{~Hz}$ or smaller, so that each data acquisition corresponds to uncorrelated speckle patterns.

\section{THEORETICAL RESULTS}

\section{A. General theory behind the setup}

In this section we present the basic theory behind the ghost imaging setup of Fig. 1. The general theory of ghost imaging has been explained in detail in Refs. [13,24]. We summarize here the main points: 
(1) The collection time of our measuring apparatus is much smaller than the time $\tau_{\text {coh }}$ over which the speckles fluctuate. Hence all the field operators are taken at equal times, and the time argument is omitted in the treatment.

(2) The speckle beam generated by the pseudo-thermal source is described by a thermal mixture, characterized by a Gaussian field statistics. Any correlation function of arbitrary order is thus expressed via the second-order correlation function

$$
\Gamma\left(\mathbf{x}, \mathbf{x}^{\prime}\right)=\left\langle a^{\dagger}(\mathbf{x}) a\left(\mathbf{x}^{\prime}\right)\right\rangle,
$$

where $a$ denotes the boson field operator of the speckle beam. Notice that we are dealing with classical fields, so that the field operator $a$ could be replaced by a stochastic $c$-number field, and the quantum averages by statistical averages over independent data acquisitions. However, we prefer to keep a quantum formalism in order to outline the parallelism with the quantum entangled beams from PDC.

(3) Information about the object is extracted by measuring the spatial correlation function of the intensities $\left\langle I_{1}\left(\mathbf{x}_{1}\right) I_{2}\left(\mathbf{x}_{2}\right)\right\rangle$, where 1 and 2 label the test and the reference beams, respectively, and $I_{i}\left(\mathbf{x}_{i}\right)$ are the operators associated to the number of photo counts over the CCD pixel located at $\mathbf{x}_{i}$ in the $i$ th beam. All the information about the object is contained in the correlation function of intensity fluctuations, which is calculated by subtracting the background term $\left\langle I_{1}\left(\mathbf{x}_{1}\right)\right\rangle\left\langle I_{2}\left(\mathbf{x}_{2}\right)\right\rangle$ :

$$
G\left(\mathbf{x}_{1}, \mathbf{x}_{2}\right)=\left\langle I_{1}\left(\mathbf{x}_{1}\right) I_{2}\left(\mathbf{x}_{2}\right)\right\rangle-\left\langle I_{1}\left(\mathbf{x}_{1}\right)\right\rangle\left\langle I_{2}\left(\mathbf{x}_{2}\right)\right\rangle .
$$

By using the input-output relations of the BS, and the standard properties of Gaussian beams, the main result obtained in Ref. [13] was

$$
\begin{aligned}
G\left(\mathbf{x}_{1}, \mathbf{x}_{2}\right)= & |r t|^{2} \\
& \times\left|\int \mathrm{d} \mathbf{x}_{1}^{\prime} \int \mathrm{d} \mathbf{x}_{2}^{\prime} h_{1}^{*}\left(\mathbf{x}_{1}, \mathbf{x}_{1}^{\prime}\right) h_{2}\left(\mathbf{x}_{2}, \mathbf{x}_{2}^{\prime}\right) \Gamma\left(\mathbf{x}_{1}^{\prime}, \mathbf{x}_{2}^{\prime}\right)\right|^{2},
\end{aligned}
$$

where $h_{1}, h_{2}$ are the impulse response function describing the optical setups in the two arms, and $r, t$ are the reflection and transmission coefficients of the BS.

Equation (3) has to be compared with the analogous result obtained for PDC beams [12]:

$G_{\mathrm{pdc}}\left(\mathbf{x}_{1}, \mathbf{x}_{2}\right)=\left|\int d \mathbf{x}_{1}^{\prime} \int d \mathbf{x}_{2}^{\prime} h_{1}\left(\mathbf{x}_{1}, \mathbf{x}_{1}^{\prime}\right) h_{2}\left(\mathbf{x}_{2}, \mathbf{x}_{2}^{\prime}\right) \Gamma_{\mathrm{pdc}}\left(\mathbf{x}_{1}^{\prime}, \mathbf{x}_{2}^{\prime}\right)\right|^{2}$,

where 1 and 2 label the signal and idler down-converted fields $a_{1}, a_{2}$, and

$$
\Gamma_{\mathrm{pdc}}\left(\mathbf{x}_{1}^{\prime}, \mathbf{x}_{2}^{\prime}\right)=\left\langle a_{1}\left(\mathbf{x}_{1}^{\prime}\right) a_{2}\left(\mathbf{x}_{2}^{\prime}\right)\right\rangle
$$

is the second-order field correlation between the signal and idler (also called biphoton amplitude).

Thus, ghost imaging with correlated thermal beams presents a deep analogy with ghost imaging with entangled beams $[13,24,25]$ : they are both coherent imaging systems, which is crucial for observing interference from a phase object, and they offer analogous performances provided that the the beams have similar spatial coherence properties. They differ in (a) the presence of $h_{1}^{*}$ at the place of $h_{1}$ (which in our case turns out to have no implications) and (b) the visibility, defined as

$$
\mathcal{V}=\max \left[\frac{G}{\left\langle I_{1} I_{2}\right\rangle}\right]=\max \left[\frac{G}{\left\langle I_{1}\right\rangle\left\langle I_{2}\right\rangle+G}\right] .
$$

In the thermal case $G\left(\mathbf{x}_{1}, \mathbf{x}_{2}\right) \leqslant\left\langle I_{1}\left(\mathbf{x}_{1}\right)\right\rangle\left\langle I_{2}\left(\mathbf{x}_{2}\right)\right\rangle$ so that the visibility is never above $\frac{1}{2}$. Conversely, in the PDC case it can be verified that $G_{\mathrm{pdc}} /\left\langle I_{1}\right\rangle\left\langle I_{2}\right\rangle$ scales as $1+\frac{1}{\langle n\rangle}$, where $\langle n\rangle$ is the mean photon number per mode (see, e.g., Ref. [13]). Only in the coincidence-count regime, where $\langle n\rangle \ll 1$, can the visibility be close to unity, while bright entangled beams with $\langle n\rangle \gg 1$ show a similar visibility as the classical beams. However, despite never being above $\frac{1}{2}$ in the classical case, we have shown $[13,23,24]$ that the visibility is sufficient to efficiently retrieve information.

The result of a specific correlation measurement is obtained by inserting into Eq. (3) the propagators describing the setup. In the case of the ghost diffraction scheme of Fig. $1, h_{1}\left(\mathbf{x}_{1}, \mathbf{x}_{1}^{\prime}\right)=(i \lambda F)^{-1} e^{-i \mathbf{x}_{1} \cdot \mathbf{x}_{1}^{\prime} k / F} T_{\mathrm{obj}}\left(\mathbf{x}_{1}^{\prime}\right)$, where $T_{\text {obj }}(\mathbf{x})$ is the object transmission function, $k=2 \pi / \lambda$, and $h_{2}\left(\mathbf{x}_{2}, \mathbf{x}_{1}^{\prime}\right)$ $=(i \lambda F)^{-1} e^{-i \mathbf{x}_{2} \cdot \mathbf{x}_{2}^{\prime} k l F}$. We get

$G\left(\mathbf{x}_{1}, \mathbf{x}_{2}\right) \propto\left|\int \mathrm{d} \mathbf{x}_{1}^{\prime} \int \mathrm{d} \mathbf{x}_{2}^{\prime} e^{i\left(\mathbf{x}_{1} \cdot \mathbf{x}_{1}^{\prime}-\mathbf{x}_{2} \cdot \mathbf{x}_{2}^{\prime}\right) k / F} T_{\mathrm{obj}}^{*}\left(\mathbf{x}_{1}^{\prime}\right) \Gamma_{\mathrm{n}}\left(\mathbf{x}_{1}^{\prime}, \mathbf{x}_{2}^{\prime}\right)\right|^{2}$

$$
=\left|2 \pi \int \mathrm{d} \mathbf{x}^{\prime} \mathcal{F}\left\{T_{\mathrm{obj}}\right\}\left[\left(\mathbf{x}_{1}-\mathbf{x}_{2}-\mathbf{x}^{\prime}\right) k / F\right] \Gamma_{\mathrm{f}}\left(\mathbf{x}_{2}, \mathbf{x}_{2}+\mathbf{x}^{\prime}\right)\right|^{2},
$$

where $\Gamma_{\mathrm{n}}$ and $\Gamma_{\mathrm{f}}$ denote the second-order field correlation function defined by Eq. (1), as measured at the object nearfield and far-field planes, respectively; $\mathcal{F}\left\{T_{\text {obj }}\right\}(\mathbf{q})$ $=\int \frac{d \mathbf{x}}{2 \pi} e^{-i \mathbf{q} \cdot \mathbf{x}} T_{\mathrm{obj}}(\mathbf{x})$ is the amplitude of the diffraction pattern from the object, and Eq. (8) is obtained from Eq. (7) with some simple passages.

We notice first of all that the result of a correlation measurement is a convolution of the diffraction pattern amplitude with the far-field coherence function $\Gamma_{\mathrm{f}}$. Hence the far-field coherence length (denoted by $\Delta x_{\mathrm{f}}$ ) determines the spatial resolution in the ghost diffraction scheme: the smaller the far-field speckles, the better resolved is the pattern. In the limit of speckles smaller than the scale of variation of the diffraction pattern, we can approximate the far-field coherence function as $\Gamma_{\mathrm{f}}\left(\mathbf{x}_{2}, \mathbf{x}_{2}+\mathbf{x}^{\prime}\right) \approx \delta\left(\mathbf{x}^{\prime}\right)\left\langle I_{\mathrm{f}}\left(\mathbf{x}_{2}\right)\right\rangle$, where $\left\langle I_{\mathrm{f}}\left(\mathbf{x}_{2}\right)\right\rangle$ is the intensity profile of the input speckle beam as observed in the far field [notice that in our ghost-diffraction setup $\left\langle I_{\mathrm{f}}\left(\mathbf{x}_{2}\right)\right\rangle=\left\langle I_{2}\left(\mathbf{x}_{2}\right)\right\rangle$, a part for some trivial proportionality factor]. In this limit we get 


$$
G\left(\mathbf{x}_{1}, \mathbf{x}_{2}\right) \propto\left|\mathcal{F}\left\{T_{\text {obj }}\right\}\left[\left(\mathbf{x}_{1}-\mathbf{x}_{2}\right) k / F\right]\right|^{2}\left\langle I_{\mathrm{f}}\left(\mathbf{x}_{2}\right)\right\rangle^{2},
$$

which means that the diffraction pattern of the object can be observed in the correlation function, when this is evaluated as a function of $\mathbf{x}_{2}$, for fixed $\mathbf{x}_{1}$. The diffraction pattern is modulated by $\left\langle I_{\mathrm{f}}\left(\mathbf{x}_{2}\right)\right\rangle^{2}$ : hence, in order to obtain the whole diffraction pattern, the far-field intensity distribution must be sufficiently broad, so that $\left\langle I_{\mathrm{f}}\left(\mathbf{x}_{2}\right)\right\rangle$ is nonzero in the region where the diffraction pattern is nonzero. It can be easily seen that this condition turns out to be equivalent to requiring spatial incoherence of the speckle beam illuminating the object, that is, the near-field coherence length (denoted here as $\Delta x_{\mathrm{n}}$ ) must be small as compared to the object scale of variation.

Equation (9) shows that the diffraction pattern can be also obtained by plotting the correlation as a function of the distance between the two points. By fixing this distance as $\mathbf{r}=\mathbf{x}_{1}-\mathbf{x}_{2}$, and varying the pixel positions in both arms as $\mathbf{x}_{1}$ and $\mathbf{x}_{2}=\mathbf{x}_{1}-\mathbf{r}$, we perform a spatial average of the correlation function, which amounts to measuring $[15,16]$

$$
\int \mathrm{d} \mathbf{x} G(\mathbf{x}, \mathbf{x}-\mathbf{r}) \propto\left|\mathcal{F}\left\{T_{\text {obj }}\right\}[\mathbf{r} k / F]\right|^{2} \int \mathrm{d} \mathbf{x}\left\langle I_{\mathrm{f}}(\mathbf{x}-\mathbf{r})\right\rangle^{2} .
$$

If the spatial average is performed over large enough regions, the integral on the right-hand side does not depend on $\mathbf{r}$ and is a constant. As already pointed out in Ref. [16], in this case there is no need of demodulating the correlation by the mean intensity in order to obtain the diffraction pattern.

Second of all, and most important for the results presented here, since the Fourier transform of the amplitude of the object transmission function is involved in Eq. (8), the imaging scheme is coherent despite the fact that the beams are incoherent. Thus, ghost diffraction of a pure phase object can be realized with spatially incoherent pseudo-thermal beams.

Quite different are the results for the HBT-type scheme, where the BS is effectively placed after the object. In this case the reference kernel changes to $h_{2}\left(\mathbf{x}_{2}, \mathbf{x}_{2}^{\prime}\right)=h_{1}\left(\mathbf{x}_{2}, \mathbf{x}_{2}^{\prime}\right)$ $=(i \lambda F)^{-1} e^{-i \mathbf{x}_{2} \cdot \mathbf{x}_{2}^{\prime} k / F} T_{\mathrm{obj}}\left(\mathbf{x}_{2}^{\prime}\right)$, and a result of correlation measurement gives

$$
G_{\mathrm{HBT}}\left(\mathbf{x}_{1}, \mathbf{x}_{2}\right) \propto\left|\int \mathrm{d} \mathbf{x}_{1}^{\prime} \int \mathrm{d} \mathbf{x}_{2}^{\prime} e^{i k / F\left(\mathbf{x}_{1} \cdot \mathbf{x}_{1}^{\prime}-\mathbf{x}_{2} \cdot \mathbf{x}_{2}^{\prime}\right)} T_{\mathrm{obj}}^{*}\left(\mathbf{x}_{1}^{\prime}\right) T_{\mathrm{obj}}\left(\mathbf{x}_{2}^{\prime}\right) \Gamma_{\mathrm{n}}\left(\mathbf{x}_{1}^{\prime}, \mathbf{x}_{2}^{\prime}\right)\right|^{2} .
$$

In the limit of spatially incoherent light $\Gamma_{\mathrm{n}}\left(\mathbf{x}_{1}^{\prime}, \mathbf{x}_{2}^{\prime}\right) \rightarrow \delta\left(\mathbf{x}_{1}^{\prime}-\mathbf{x}_{2}^{\prime}\right)\left\langle I_{n}\left(\mathbf{x}_{1}^{\prime}\right)\right\rangle$, and Eq. (11) can be recast as

$$
G_{\mathrm{HBT}}\left(\mathbf{x}_{1}, \mathbf{x}_{2}\right) \propto\left|\int \mathrm{d} \mathbf{x}^{\prime} \mathcal{F}\left\{\left|T_{\mathrm{obj}}\right|^{2}\right\}\left[\left(\mathbf{x}_{2}-\mathbf{x}_{1}+\mathbf{x}^{\prime}\right) k / F\right] \Gamma_{\mathrm{f}}\left(\mathbf{x}_{2}, \mathbf{x}_{2}+\mathbf{x}^{\prime}\right)\right|^{2} .
$$

By comparing with the result of Eq. (8) for ghost diffraction, we see that the HBT scheme only gives information about the Fourier transform of the modulus squared of the object transmission function: in the limit of incoherent light the imaging scheme is incoherent. Thus, the phase information about the object is lost and the HBT scheme is able to see interference only from absorptive objects.

We can now consider the opposite limit, of spatially coherent light illuminating the object, achieved when the coherence length $\Delta x_{\mathrm{n}}$ (the speckle size) at the object plane is large compared to the object size. In this case, the HBT scheme allows us to retrieve the diffraction pattern even of a pure phase object [34]. In this limit, in fact, the coherence function $\Gamma_{\mathrm{n}}$ can be taken as roughly constant over the regions of integration in Eq. (11), which hence gives

$$
G_{\mathrm{HBT}}\left(\mathbf{x}_{1}, \mathbf{x}_{2}\right) \propto\left|\mathcal{F}\left\{T_{\text {obj }}\right\}\left(\mathbf{x}_{1} k / F\right)\right|^{2} \times\left|\mathcal{F}\left\{T_{\text {obj }}\right\}\left(\mathbf{x}_{2} k / F\right)\right|^{2} .
$$

Evidently, if we fix $\mathbf{x}_{1}$ and evaluate the correlation as a function of $\mathbf{x}_{2}$ we observe the diffraction pattern, even of a pure phase object.

Notably, in the ghost diffraction scheme no diffraction pattern appears in the correlation as a function of the refer- ence pixel position $\mathbf{x}_{2}$ for spatially coherent light. In the limit of spatial coherence, Eq. (7) factorizes into the product of two integrals, one showing the diffraction pattern of the object in arm 1, as a function of $\mathbf{x}_{1}$, the other showing the mean far-field intensity profile in arm 2 . This can be readily seen also from Eq. (8), where the limit of spatially coherent light at the object plane (limit of a single large speckle illuminating the object) amounts to $\left\langle I_{\mathrm{f}}\left(\mathbf{x}_{2}\right)\right\rangle \rightarrow \delta\left(\mathbf{x}_{2}\right)$.

In practice, the classic HBT scheme uses the cross correlation of two beams split on a BS after the object as a convenient way of measuring the autocorrelation of the beam transmitted through the object, as, e.g., done in Ref. [7]. We will actually measure the autocorrelation of the light in the test arm in the focal plane of the lens, defined as

$$
C_{\text {auto }}\left(\mathbf{x}, \mathbf{x}^{\prime}\right)=\left\langle I_{1}(\mathbf{x}) I_{1}\left(\mathbf{x}^{\prime}\right)\right\rangle-\left\langle I_{1}(\mathbf{x})\right\rangle\left\langle I_{1}\left(\mathbf{x}^{\prime}\right)\right\rangle .
$$

Apart from a small shot-noise contribution at $\mathbf{x}^{\prime}=\mathbf{x}$ and some irrelevant proportionality factors, the results expected from such a measurement coincide with those of the HBT schemes described by Eq. (11), and in the proper limits by Eqs. (12) and (13).

This comparison between the HBT and the ghost diffraction schemes indicates that the measurement of the autocor- 
relation function is a valid test to prove whether or not a given object alters the phase of the incoming light or not: in the presence of spatially incoherent light, the ghost diffraction scheme fully preserves the information about the object phase in the diffraction pattern, whereas this information is lost in the autocorrelation. For a pure phase object, no interference pattern at all should appear in the autocorrelation. However, as it will become clearer in the next sections, the autocorrelation is extremely sensitive to the degree of spatial incoherence in the beam: even a small partial coherence in the incoming beam is sufficient to preserve some phase information in the autocorrelation function.

We finally stress that despite having discussed the ghost diffraction results in the framework of classical speckle light [i.e., for which Eq. (3) holds], the results (8)-(13) hold also for the entangled case [for which Eq. (5) holds].

\section{B. The object}

We have chosen a commercially available TGBS as our pure phase object. It is well known that such a device transmits the incoming light (and has close to zero absorption/ reflection) so that in the far zone of the object several distinct peaks are observed. This is because the diffraction angle obeys the thin grating equation

$$
\sin \left(\theta_{n}\right)=n \lambda / d, \quad n=0, \pm 1, \pm 2, \ldots .
$$

This equation holds when the incoming light is a plane wave normally incident on the grating, and tells that the light is observed in several orders $n$ of the diffraction angle $\theta_{n}$, and that the location of the diffraction peaks is found according to the ratio $\lambda / d$ where $\lambda$ is the wavelength of the light and $d$ is the period of the grating.

When observing the intensity distribution of the transmitted light in the far zone, the strength of the $n$th diffraction peak is $\eta_{n}=\left|c_{n}\right|^{2}$, where $c_{n}$ are the diffraction coefficients. Since a grating can be thought of as an infinite repetition of a single period of the grating $T_{\mathrm{obj}}$, we may use Fourier series theory to write the diffraction coefficients [31]

$$
c_{n}=\frac{1}{d} \int_{0}^{d} d x T_{\mathrm{obj}}(x) \exp (-i 2 \pi n x / d) .
$$

A TGBS is a grating made of a completely transparent material which has grooves cut on the exit side so that a phase difference is imposed on the field exiting the grating depending on the position. This phase difference can be expressed through the groove depth $\delta$ as $\Delta \phi=\left(n_{g}-1\right) 2 \pi \delta / \lambda$, where $n_{g}$ is the refractive index of the grating material. Thus, $\delta$ can be chosen to give the desired phase shift.

Typically, square gratings of width $a$ (and period $d$ ) are used for TGBS, so that within the period $d$ the object transmission function is

$$
T_{\text {obj }}(x)=\left\{\begin{array}{cc}
e^{i \Delta \phi}, & 0 \leqslant x \leqslant a, \\
1, & a<x \leqslant d .
\end{array}\right.
$$

Calculating the diffraction coefficients gives

$$
\eta_{0}=1+\sin ^{2}(\Delta \phi / 2) \frac{4 a}{d}(a / d-1),
$$

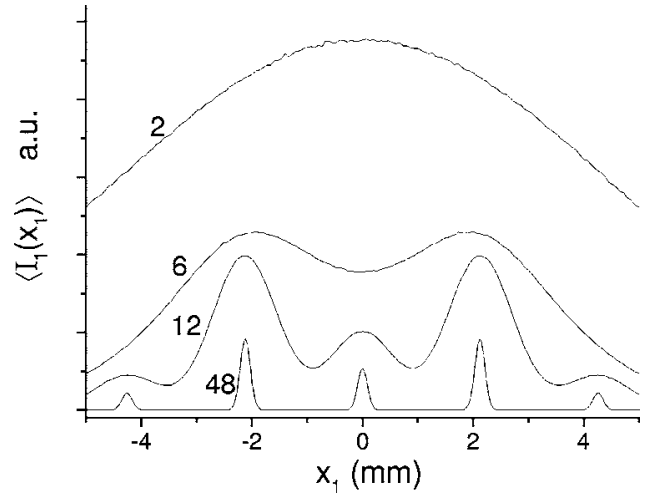

FIG. 2. Numerical simulations demonstrating the transition between coherent and incoherent illumination of the object. Average intensity $\left\langle I_{1}\right\rangle$ in the far field of the test arm for different values of the speckle size at the object plane $\Delta x_{\mathrm{n}}=2,6,12$, and $48 \mu \mathrm{m} \mathrm{Nu}-$ merical parameters: we use 1024 grid points, and 32 pixels per groove period; $d=12.5 \mu \mathrm{m} ; a=4.2 \mu \mathrm{m}, \Delta \phi=0.84 \pi ; \Delta x_{\mathrm{f}}=80 \mu \mathrm{m}$; averages are done over $10^{5}$ independent realizations.

$$
\eta_{n}=\sin ^{2}(\Delta \phi / 2) \frac{4 a^{2}}{d^{2}} \operatorname{sinc}^{2}(\pi n a / d), \quad n= \pm 1, \pm 2, \ldots
$$

Choosing $\Delta \phi$ and the ratio $a / d$ properly one can engineer the peaks to have the desired distribution.

Our TGBS is from Edmund Optics and has 80 grooves per mm (stock no. NT46-069), i.e., $d=12.5 \mu \mathrm{m}$. It is designed for $\lambda=633 \mathrm{~nm}$ to have $25 \%$ of the power in the 0 and \pm 1 peaks and $5 \%$ in the \pm 2 order peaks. This means $\eta_{0}=\eta_{1}$ and $\eta_{1} / \eta_{2}=5$, implying that $\Delta \phi=0.71 \pi$ and that $a / d=\arccos (1 / \sqrt{5}) / \pi, \quad$ giving $a=d / 2.84=4.4 \mu \mathrm{m}$. The smallness of $a$ sets a limit for the relative coherence of the object beam, as discussed in detail in what follows.

Since we are using a frequency doubled Nd:YAG laser with $\lambda=532 \mathrm{~nm}$ the phase difference is instead $\Delta \phi=\frac{633}{532} 0.71 \pi \simeq 0.84 \pi$. This implies $\eta_{1} / \eta_{0}=2.2$, i.e., the central peak should be roughly a factor of 2 weaker than the \pm 1 order peaks when using light at this wavelength.

\section{Preliminary investigation through numerics}

In this section we will discuss some numerical results we used to select the size of the speckles we would need in the object plane in order to render the beam spatially incoherent. The numerical simulations were done using the method described in Ref. [24]. Essentially, we Fourier transform noise convoluted with a Gaussian to obtain a certain speckle size in the object plane $\Delta x_{\mathrm{n}}$ (the size of which is controlled by the waist of the Gaussian). Imposing a pinhole of size $D_{\mathrm{ph}}$ on this field and performing another Fourier transform gives a speckle field as observed in the far field of the object plane; the speckle size there, $\Delta x_{\mathrm{f}}$, is determined by $D_{\mathrm{ph}}$ and is therefore controlled independently of $\Delta x_{\mathrm{n}}$. The object used in the simulations was a purely transmissive square grating chosen to mimic the object predicted theoretically in Sec. III B (see Fig. 2 for specific parameters). Since ghost imaging implies that coherent imaging is done with incoherent light, it is 


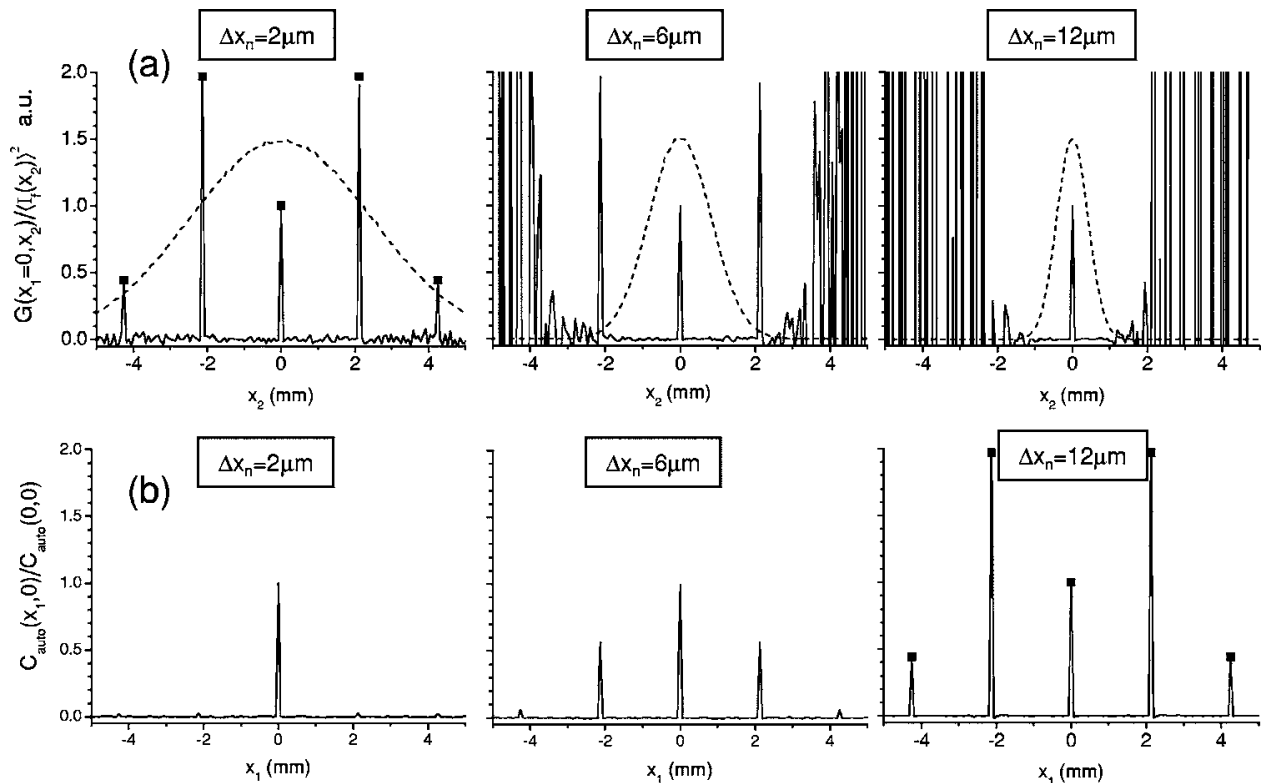

FIG. 3. Numerical simulations demonstrating the transition between coherent and incoherent illumination of the object. (a) The cross correlation (solid line) normalized by the square of the mean intensity in the reference arm (the latter is shown by the dotted line) as obtained for different sizes $\Delta x_{\mathrm{n}}$ of the near-field speckles. (b) The corresponding autocorrelation functions of the test arm intensity. The black squares indicate the peak values of the analytical diffraction pattern. Parameters: as in Fig. 2. important that the light is really incoherent relative to the object details. Thus, in a direct observation of the test arm far-field average intensity, $\left\langle I_{1}\right\rangle$, we should not be able to see the diffraction pattern of the object. As shown in Fig. 2, when $\Delta x_{\mathrm{n}} \approx 2 \mu \mathrm{m},\left\langle I_{1}\right\rangle$ does not reveal any information about the diffraction pattern. Thus, such a speckle size corresponds to practically incoherent illumination of the object. However, as the speckle size is increased $\left(\Delta x_{\mathrm{n}}=12 \mu \mathrm{m}\right),\left\langle I_{1}\right\rangle$ reveals more and more information about the diffraction pattern, corresponding to partially coherent illumination. For large speckle sizes $\left(\Delta x_{\mathrm{n}}=48 \mu \mathrm{m}\right)\left\langle I_{1}\right\rangle$ is very close to the analytical diffraction pattern and the illumination is close to being completely coherent [35].

As shown in Sec. III A, if the test beam is incoherent with respect to the object, the autocorrelation of $I_{1}$ should not reveal at all the diffraction pattern of a pure phase object, while the pattern should appear in the cross correlation. Figure 3 compares the cross correlation function $G\left(\mathbf{x}_{1}, \mathbf{x}_{2}\right)$ and the autocorrelation $C_{\text {auto }}\left(\mathbf{x}_{1}, \mathbf{x}_{1}^{\prime}\right)$ of the test arm intensity, for different sizes of the near-field speckles. Both functions are calculated for a fixed $\mathbf{x}_{1}$. For $\Delta x_{n}=2.0 \mu \mathrm{m}$ the cross correlation shows a diffraction pattern that, as we verified, is very close to the pattern analytically calculated. On the contrary, the autocorrelation shows very little information in the sidebands; the $n=1$ sideband is $2.4 \%$ of the central $(n=0)$ peak. This confirms that this speckle size corresponds to practically incoherent illumination. The fact that some information is observed in the sidebands at all is because the $2.0 \mu \mathrm{m}$ speckle on the scale of the object is not vanishingly small, but merely small. By increasing the coherence of the light, we notice that the object information disappears from the cross correlation and appears in the autocorrelation, in agreement with Eq. (13) and with the discussion in Sec. III A. For $\Delta x_{n}=12 \mu \mathrm{m}$ the sidebands present in the autocorrelation coincide almost completely with the analytical diffraction pattern, showing thus that the autocorrelation function is sensitive to the presence of even a small partial coherence of the light.
To conclude this discussion, we have to choose a speckle size at the object plane around $2 \mu \mathrm{m}$ in order to have beams that are truly incoherent with respect to the object details, so that information about the object is revealed neither in the autocorrelation nor the average of the test beam far-field intensity.

\section{EXPERIMENTAL RESULTS}

\section{A. Pseudo-thermal source and speckles from near-field scattering}

As shown in the previous section, the physical size of the TGBS requires a speckle size at the object plane $\Delta x_{\mathrm{n}}$ less than the finest object detail, i.e., less than $4.4 \mu \mathrm{m}$. Such a small size is not easy to achieve, but we managed to create speckles with $\Delta x_{\mathrm{n}}=2.0 \mu \mathrm{m}$ by placing the object very close to our pseudo-thermal source $(z=18 \mathrm{~mm}$, practically limited only by the physical size of the half-inch cube BS). The speckles generated this way are the so-called near-field speckles (NFSs) [29,30], which are remarkably different from the classical far-field speckles (FFSs), whose size is determined by the well-known Van Cittert-Zernike theorem [32].

This part of the setup is quite different from what we used in our previous experiments in Refs. [23-25] where the object was in the far zone of the source. Instead, in the current setup the source is very close to the object, and, as explained in detail below, at a given point of the object plane the waves interfering do not originate from the entire illuminated spot.

To understand how NFS are formed, let us first describe the pseudo-thermal source in some detail.

Our thermal source consists of a laser beam illuminating a slowly rotating ground glass, followed by a square cell $5 \mathrm{~mm}$ thick, containing a concentrated solution of latex particles with average diameter $\rho=3 \mu \mathrm{m}$. The cell is almost in physical contact with the rotating glass and on its exit face there is a pinhole (diameter $D_{\mathrm{ph}}=4.5 \mathrm{~mm}$ ) which determines the transverse size of the source. The combination of the ground 
glass and the turbid solution is an easy and convenient way to generate truly random speckles. Indeed, the ground glass alone would produce only partially stochastic speckles because the pattern would be reproduced after one turn of the glass. On the other hand, the turbid cell guarantees stochasticity, but if used alone would exhibit a residual transmitted coherent component which is clearly undesired.

Due mainly to Brownian particle motion (and secondarily to glass rotation), the speckle pattern generated by the source fluctuates randomly with time and is characterized by a coherence time $\tau_{\text {coh }}$ which can be tuned by varying the turbidity of the solution. In our case we had $\tau_{\mathrm{coh}} \approx 10 \mathrm{~ms}$.

Multiple scattering occurs inside the cell, so that the light beam exiting the source has a divergence angle $\Theta_{\text {eff }}$ larger than the angle that would be expected from single scattering. The latter is associated to particle diffraction and given by $\Theta_{\text {dif }} \approx \lambda / \rho$. The effective value of $\Theta_{\text {eff }}$ depends on the detailed features of the scattering cell, as particle concentration and cell length; in practice, we can claim that our thick thermal source behaves as an ideal thin thermal source characterized by spatial inhomogeneities or "scatterers" of effective diameter $\rho_{\text {eff }} \approx \rho\left(\Theta_{\mathrm{dif}} / \Theta_{\mathrm{eff}}\right)<\rho$.

When the light generated by such a source is observed at a plane located at a sufficiently large distance $z$, each point of this plane is reached by the contributions emerging from the entire radiating region $D_{\mathrm{ph}}$. Under this condition, the stochastic interference between the (many) different waves gives rise to a specklelike intensity distribution (far-field speckle, FFS), whose correlation function is described by the Van Cittert-Zernike theorem, and is characterized by the average speckle size [32]

$$
\Delta x \approx z \frac{\lambda}{D_{\mathrm{ph}}} \quad(\mathrm{FFS})
$$

Thus, the requirement for obtaining FFS is $\Theta_{\text {eff }} z \gg D_{\text {ph }}$, i.e.,

$$
z \gg \frac{D_{\mathrm{ph}} \rho_{\mathrm{eff}}}{\lambda} \quad(\mathrm{FFS})
$$

When this condition is not fulfilled, the waves interfering at each point of the observation plane originate from a region $D^{*} \approx \Theta_{\text {eff }} z \approx \frac{\lambda}{\rho_{\text {eff }}} z$ smaller than the radiating region $D_{\mathrm{ph}}$. Provided that $D^{*}$ is not too small, at a given point in the observation plane one would still get contributions from many different scatterers, which is sufficient to produce near-field speckles. Applying again the Van-Cittert Zernike theorem with $D^{*}$ instead of $D_{\mathrm{ph}}$, one gets the remarkable result [29]

$$
\Delta x \approx \rho_{\mathrm{eff}} \quad(\mathrm{NFS}),
$$

according to which the average NFS size is only determined by the effective size of the scatterers and is independent of both $\lambda$ and $z$. To fulfill the criteria for NFS we must have $(i)$ $D^{*}<D$ and (ii) many scatterers inside $D *$, e.g., $\left(D^{*} / \rho_{\text {eff }}\right)^{2}$ $\gg 1$. This implies that the distance $z$ must fulfill the two conditions:

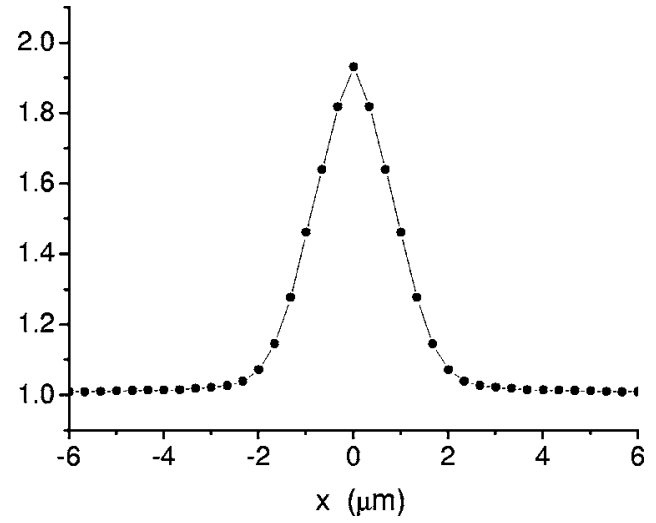

FIG. 4. Spatial autocorrelation function of the speckle beam just before the object $\left\langle I_{\mathrm{n}}\left(\mathbf{x}^{\prime}\right) I_{\mathrm{n}}\left(\mathbf{x}^{\prime}+\mathbf{x}\right)\right\rangle$. The peak above the baseline has a FWHM of $1.98 \pm 0.02 \mu \mathrm{m}$, which gives the characteristic size of the near-field speckles.

$$
z \ll \frac{D_{\mathrm{ph}} \rho_{\mathrm{eff}}}{\lambda}, \quad z \gg \frac{\rho_{\mathrm{eff}}^{2}}{\lambda} \quad(\mathrm{NFS}) .
$$

In our experiment we have $\lambda=0.532 \mu \mathrm{m}, D_{\mathrm{ph}}=4.5 \mathrm{~mm}$, and $\rho_{\text {eff }} \leqslant 3 \mu \mathrm{m}$. Thus the second criterium is easily fulfilled because $\frac{\rho_{\text {fff }}^{2}}{\lambda}<\frac{\rho^{2}}{\lambda}=17 \mu \mathrm{m}$, so that $z \gg 17 \mu \mathrm{m}$ is enough. The first criterium is somehow more difficult to evaluate, because it requires more detailed knowledge of $\rho_{\text {eff }}$. However, our final purpose was to make speckles as small as possible and, therefore, we set the distance between the object and the pseudo-thermal source to its minimum value $z=18 \mathrm{~mm}$ [36], which to a large extent meets the first criterium in Eq. (23).

Then we measured the speckle pattern in the object plane by removing the lens $F$ and inserting a $\times 20$ objective to image the object plane on the CCD (the magnification is needed because the CCD pixel size is $6.7 \mu \mathrm{m}$ ). The speckle size was finally estimated by performing the autocorrelation of such a pattern as shown in Fig. 4. The peak above the baseline had a full width at half-maximum (FWHM) of $1.98 \pm 0.02 \mu \mathrm{m}$. This gives an estimate of the near-field speckle size (see Refs. [23,24] for more details) $\Delta x_{\mathrm{n}}$ $\approx 1.98 \mu \mathrm{m}$, which, as shown in Sec. III C, should be small enough to render the beams incoherent with respect to the object details.

For completeness, we also measured the size of the speckles in the far field (the size of the speckles on the CCD) by removing the objective and reinserting the lens $F$. The procedure gave $\Delta x_{\mathrm{f}}=11.1 \pm 0.1 \mu \mathrm{m}$. This value actually overestimates the real size of the far-field speckles, because the CCD pixel size $(6.7 \mu \mathrm{m})$ is too large with respect to the speckles, so that the speckle pattern undergoes a substantial smoothing. In any case, the measured $\Delta x_{\mathrm{f}}$ determines the spatial resolution of the ghost diffraction pattern, and in our case turns out to determine the width of the ghost diffraction peaks.

\section{B. Ghost diffraction versus HBT scheme: Case of incoherent illumination}

We performed a first set of measurements keeping the object plane at a distance $z=18 \mathrm{~mm}$, thus having speckles at the object plane of size $\Delta x_{\mathrm{n}} \approx 2 \mu \mathrm{m}$. 
In order to characterize the diffraction pattern created by the TGBS and to provide a reference for the ghost diffraction pattern, we performed preliminary measurements with coherent laser light: we removed the scattering media from the setup of Fig. 1 and recorded the transmitted light of the TGBS in the focal plane of the lens $F$. This measurement was already not straightforward because of the large values of the scattering angles of the grating equation (15): the $n$ th-order peaks at angles $\theta_{n}=n \lambda / d$ are displaced in the far field at positions $x_{n}=\theta_{n} F=n F \lambda / d$. Using the numbers in our setup we have $x_{ \pm 1}= \pm 2.13 \mathrm{~mm}$ and $x_{ \pm 2}= \pm 4.26 \mathrm{~mm}$, so that the distance between the two second-order peaks is larger than the extension of our CCD. We therefore had to do three measurements in order to observe all the peaks: first $n=-2$, -1 , and 0 were observed, then the CCD was shifted to observe $n=-1,0$, and +1 , and finally $n=0,+1$, and +2 . A second problem was represented by the small width of the diffraction peaks (few pixels on the CCD) which provided a too poor sampling of the diffraction pattern. In order to evaluate the relative heights of the peaks we performed an integration in the region around each peak, which gave $\eta_{1} / \eta_{0}=4$ and $\eta_{1} / \eta_{2}=2$. Moreover, the diffraction pattern is not symmetric and, for example, $\eta_{-1} \neq \eta_{1}$. This is somewhat different from what we expected from the theory and probably depends on some defects of fabrication of the TGBS, but it will serve as our reference. Experimentally we found the peaks to be located at $x_{ \pm 1}= \pm 2.22 \mathrm{~mm}$ and $x_{ \pm 2}= \pm 4.43 \mathrm{~mm}$, in good agreement with the theory.

We also used the coherent illumination to set the origin of our coordinate systems: in the test $\operatorname{arm} \mathbf{x}_{1}=0$ corresponds to the the $n=0$ diffraction peak, while in the reference arm the $\mathbf{x}_{2}=0$ point is the location of the reference beam. If the testarm pixel is for example fixed at $\mathbf{x}_{1}=0$ in the subsequent correlation measurements, we expect that the ghost diffraction pattern will emerge in a region of the reference arm centered around $\mathbf{x}_{2}=0$, while by shifting $\mathbf{x}_{1}$ the pattern will shift accordingly.

We then reinserted the scattering media to measure the ghost diffraction pattern of the TGBS from the cross correlation between the two arms. A typical snapshot of what we observe on the CCD is shown in Fig. 5(a). The upper part contains the reference arm intensity. For the correlation, we selected a narrow strip (128 pixel wide) centered around $y$ $=0$ and extending over the entire $x$ axis (1024 pixels). In the test arm no spatial information was extracted since there we collected the light from a single fixed pixel. Initially we located the pixel in the test arm at the point $\mathbf{x}_{1}=0$ [pixel at position $P_{2}$ in Fig. 5(a)], and we measured the cross correlation $\left\langle I_{1}\left(\mathbf{x}_{1}=P_{2}\right) I_{2}\left(\mathbf{x}_{2}\right)\right\rangle$ as a function of $\mathbf{x}_{2}$ varying in the region shown by the white frame in Fig. 5(a), by averaging over 18000 snapshots. As seen from Eq. (9) this gives a diffraction pattern that is centered on $\mathbf{x}_{2}=0$. In this case we are able to reconstruct only the $n=-1,0$, and +1 peaks, because the higher order peaks are outside of the reference region imaged on the $\mathrm{CCD}$. In order to reconstruct also the $n= \pm 2$ peaks, we repeated twice the measurement by shifting the test arm pixel at the positions $P_{1}$ and $P_{3}$, respectively [see Fig. 5(a)], so that the diffraction pattern emerging from the correlation shifts accordingly, as dictated by Eq. (9).

The results of these measurements are shown in Fig. 5(b), which plots the cross correlation scaled to $\left\langle I_{\mathrm{f}}\left(\mathbf{x}_{2}\right)\right\rangle^{2}$. The 2-D (a)
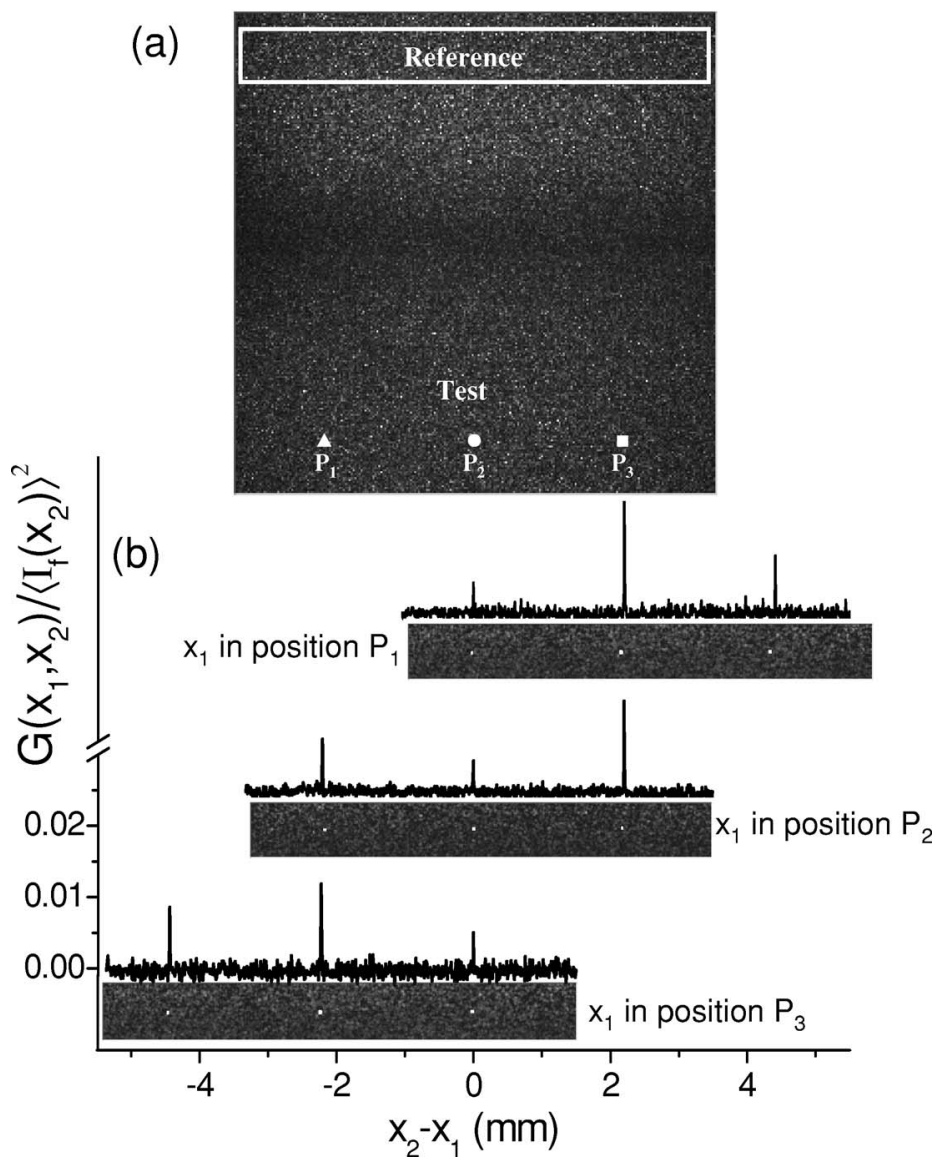

FIG. 5. Experimental demonstration of ghost diffraction of a pure phase object using incoherent classical light. (a) Snapshot of the speckles recorded by the CCD in the far-field plane. The reference beam is in the upper region and the white frame shows the region used for the correlation. The test arm is in the lower region, and the white symbols indicate the three different single-pixel positions used for the correlation. (b) Ghost diffraction patterns reconstructed via the cross correlation between the test and the reference arm, measured by locating $\mathbf{x}_{1}$ at each of the three pixel positions, and by varying $\mathbf{x}_{2}$ (18 000 averages). The 2-D plots of the cross correlation $G\left(\mathbf{x}_{1}, \mathbf{x}_{2}\right) /\left\langle I_{\mathrm{f}}\left(\mathbf{x}_{2}\right)\right\rangle^{2}$ are shown as functions of $\mathbf{x}_{2}-\mathbf{x}_{1}$, together with their 1-D cut in the horizontal direction.

reconstructed diffraction patterns are shown close to their cut along the $x$ direction, for each positioning of $\mathbf{x}_{1}$. Since each diffraction peak covers only few pixels, the $x$ cuts of Fig. 5 (b) only give a qualitative image of the diffraction pattern, but do not allow a quantitative estimation of the peak heights, due to the poor sampling. In order to extract the relative height of the peaks, we located groups of pixels having a substantial value above the noise floor and added together their values, which effectively corresponds to integrating over each peak. The results are shown in Fig. 6 and compared with those obtained from coherent laser illumination with the same technique. We observe that they agree extremely well: we have successfully created the correct diffraction pattern of the pure phase object from the correlation. Also notice that the $n= \pm 1$ peaks are recorded twice, and the $n=0$ is recorded three times: these overlaps happen when displacing the single pixel position, and they agree very well 


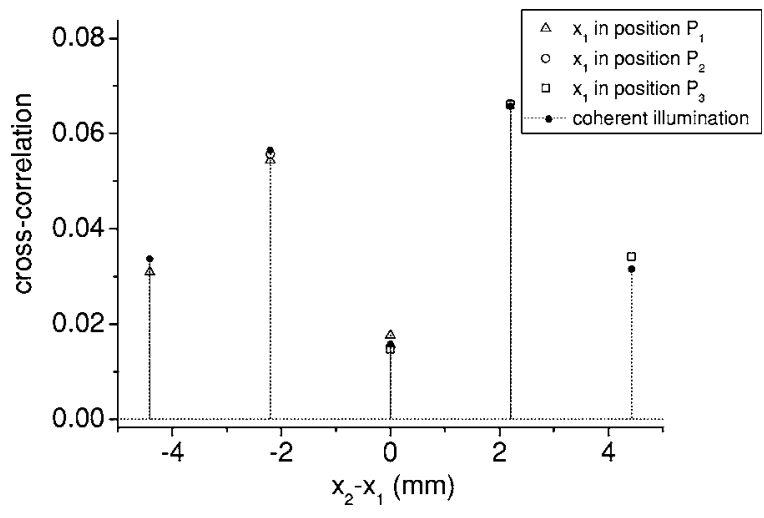

FIG. 6. Quantitative comparison between ghost diffraction and laser illumination. The height of the diffraction peaks of the cross correlation shown in Fig. 5 are evaluated by performing an integral over the peaks. They are compared with peak values measured via coherent laser illumination.

with each other. Finally, note that the position of the diffraction peaks agree well with the theoretical prediction in Sec. III B.

As discussed in Sec. III A, the spatial average technique provides a a faster and more efficient way of measuring the ghost diffraction pattern. In this case the cross correlation is measured by varying both $\mathbf{x}_{2}$ and $\mathbf{x}_{1}$ for a fixed $\mathbf{x}_{2}-\mathbf{x}_{1}$. The results of this kind of measurement are shown in Fig. 7, where the upper part of the figure is a 2-D plot of the measured cross correlation, and the lower part displays the integral over the diffraction peaks compared to the laser illumination results. Also in this case the agreement is excellent. From this figure we notice that only few averages over snapshots are needed to reconstruct the diffraction pattern, because a large number of averages over spatial points is performed, thus increasing the convergence rate $[15,16]$. Moreover the whole diffraction pattern is reconstructed in a single measurement. Despite being much more efficient than the single-pixel reconstruction, the spatial average technique

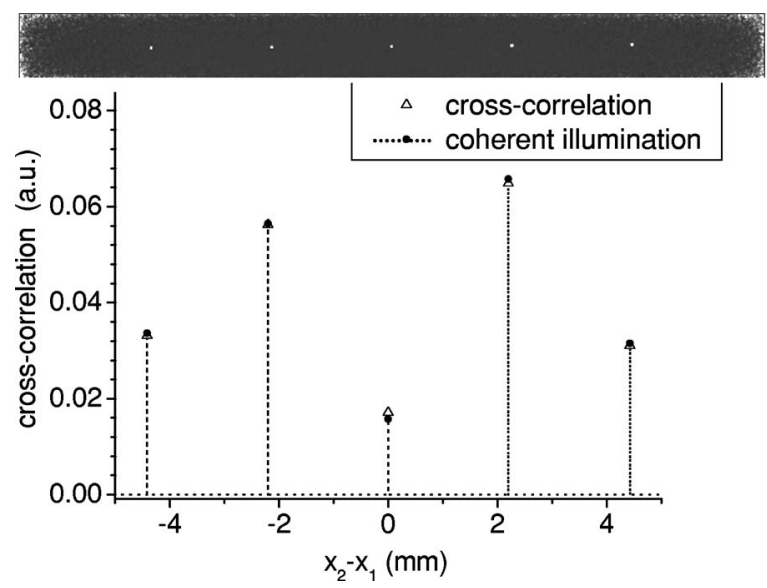

FIG. 7. Cross-correlation function $\left\langle I_{2}\left(\mathbf{x}_{2}\right) I_{1}\left(\mathbf{x}_{1}\right)\right\rangle$, calculated with the spatial average technique. The upper part is a $2 \mathrm{D}$ plot of the correlation function (100 averages). The lower plot displays the integrals over the peaks compared with the results of laser illumination.

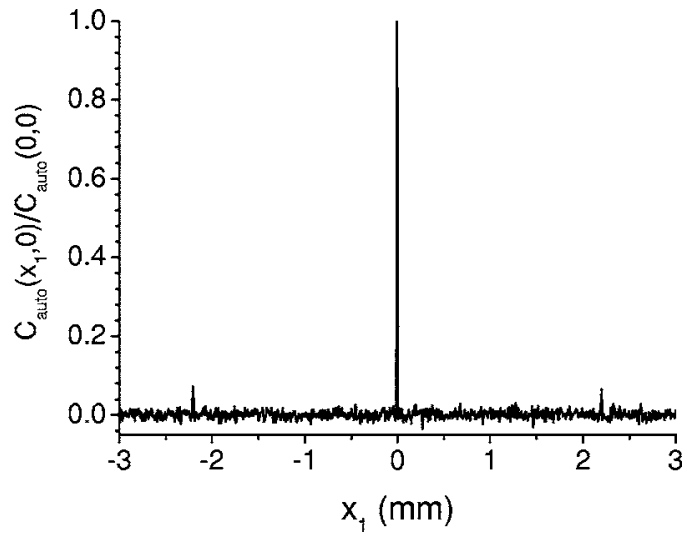

FIG. 8. Autocorrelation function $\left\langle I_{1}\left(\mathbf{x}_{1}\right) I_{1}\left(\mathbf{x}_{1}^{\prime}\right)\right\rangle$ of the test arm, measured by fixing $\mathbf{x}_{1}^{\prime}$ at position $P_{2}$ and varying $\mathbf{x}_{1}(10000$ averages).

does not follow the ghost imaging original spirit, which assumes that the imaging information is extracted by only operating on the reference arm. In this case, spatial information is also extracted from the test beam 1 , by varying the pixel $\mathbf{x}_{1}$.

As a straightforward demonstration of the degree of incoherence of the beams used, we present in Fig. 8 a measure of the autocorrelation function in the test $\operatorname{arm} C_{\text {auto }}\left(\mathbf{x}_{1}, \mathbf{x}_{1}^{\prime}\right)$. This is measured by fixing $\mathbf{x}_{1}^{\prime}$ at position $P_{2}$ and by varying $\mathbf{x}_{1}$, in the same way as described in Sec. III C.

Evidently it does not reveal any significant information about the diffraction pattern. In fact, the first-order peaks are barely visible and are at a level of $8 \%$ of the main peak, in trend with the prediction of the numerical results of Sec. III C. As argued in Sec. III A, this type of measurement is equivalent to a HBT-type scheme, and it works as an incoherent imaging scheme when using incoherent light; in this case it is expected to give no information about a pure phase object. We can thus conclude that $(i)$ the TGBS is truly a pure phase object and (ii) the speckle light we use is truly incoherent relative to the object.

\section{Ghost diffraction versus HBT schemes: Case of partially coherent illumination}

In this section we present results obtained by gradually increasing the spatial coherence of the light illuminating the object. This is achieved by increasing the distance $z$ between the pseudo-thermal source and the object plane (see Fig. 1).

We performed a second set of measurements with this distance set as $z=115 \mathrm{~mm}$. The measured autocorrelation of the light illuminating the object gave a speckle size $\Delta x_{\mathrm{n}}=14 \mu \mathrm{m}$ (FWHM of the autocorrelation peak). The main results obtained in these conditions are displayed in Fig. 9. In a third set of measurements the object-source distance was $z=300 \mathrm{~mm}$ and the measured speckle size was $\Delta x_{\mathrm{n}}=33 \mu \mathrm{m}$. Figure 10 displays the results in this case.

By increasing the source-object distance the light gains some partial coherence relative to the object. This is already evident by the distribution of the speckles recorded in the far field of the test arm, shown in the lower parts of frames (a) in 
(a)

$$
\Delta x_{n}=14 \mu m
$$

(a)
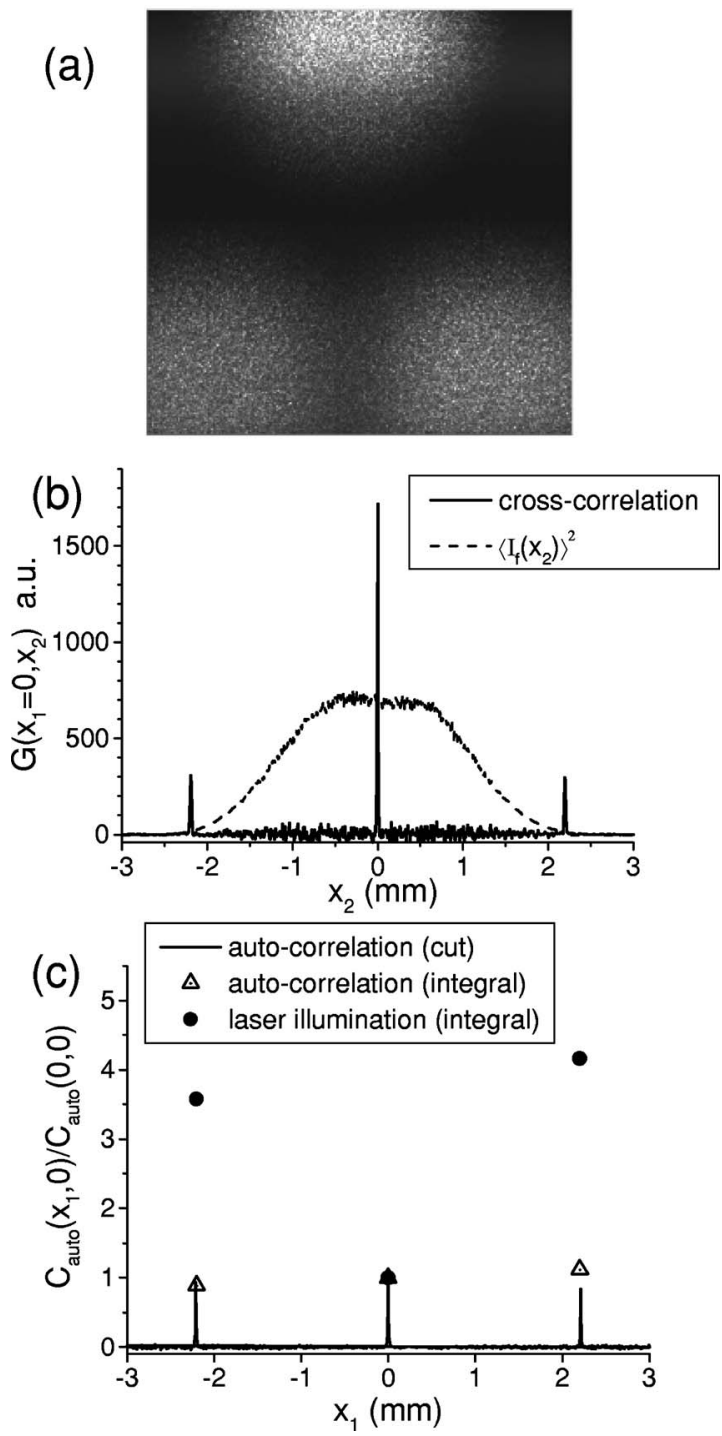

FIG. 9. Case of partially coherent illumination. The distance source-object is $z=115 \mathrm{~mm}$, with $\Delta x_{\mathrm{n}}=14 \mu \mathrm{m}$. (a) Far-field speckle distribution in a single snapshot (upper part: reference arm, lower part: test arm). (b) Cross correlation of the test and reference arm, for $\mathbf{x}_{1}$ fixed at the origin and 30000 averages. The dashed curve shows the reference intensity squared. (c) Autocorrelation of the test arm light intensity. Full line: horizontal section of the autocorrelation function. Open triangles: peak values of the autocorrelation (integral over the peaks). Circles: peak values measured with coherent laser illumination.

Figs. 9 and 10. Differently from the case of incoherent illumination, where the mean intensity distribution of the test arm is almost flat [see Fig. 5(a)], two broad peaks in correspondence to the $n= \pm 1$ diffraction orders are now clearly distinguishable in the speckle distribution. As the coherence of the light increases [Fig. 10(a)] they become narrower and more pronounced. Notice that the zeroth-order peak is barely visible in these plots because its intensity is lower (as dictated by the TGBS).

The cross correlation between the test and the reference arm, obtained by fixing $\mathbf{x}_{1}$ at position $P_{2}$, is plotted in frames
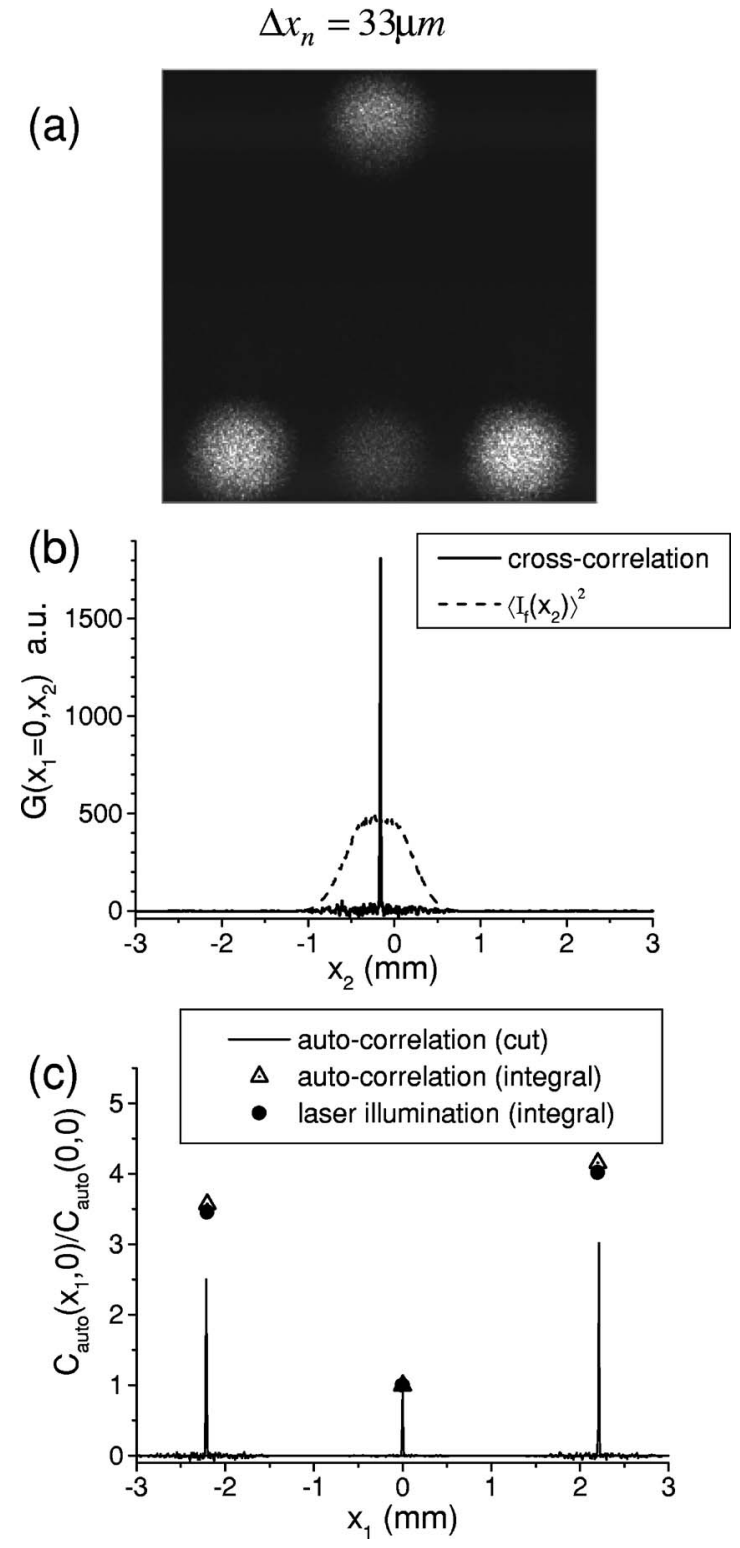

FIG. 10. The spatial coherence of the light illuminating the object is further increased: $z=300 \mathrm{~mm}, \Delta x_{\mathrm{n}}=33 \mu \mathrm{m}$. Frames (a)-(c) display the same quantities as in Fig. 9.

(b) of Figs. 9 and 10. We see that by increasing the coherence of the light, the height of the \pm 1 diffraction peaks decreases with respect the zeroth-order peak, and the diffraction pattern gradually disappears from the cross correlation. Notice that these plots shows the "bare" cross-correlation function $G\left(\mathbf{x}_{1}, \mathbf{x}_{2}\right)$ [i.e., there is no scaling factor $\left.\left\langle I_{\mathrm{f}}\left(\mathbf{x}_{2}\right)\right\rangle^{2}\right]$. As predicted by Eq. (9), the correlation scales with the square of the mean intensity of the reference arm, whose profile is plotted by the dashed lines in the figures. By increasing the near-field coherence, the far-field intensity spot becomes narrower, until the mean intensity vanishes in the region where the higher order diffraction peaks should emerge. In principle, the correct height of the diffraction peaks could be recovered by dividing the correlation by $\left\langle I_{\mathrm{f}}\left(\mathbf{x}_{2}\right)\right\rangle^{2}$, but this operation also amplifies the noise in the regions where the intensity level is low. This is evident when 

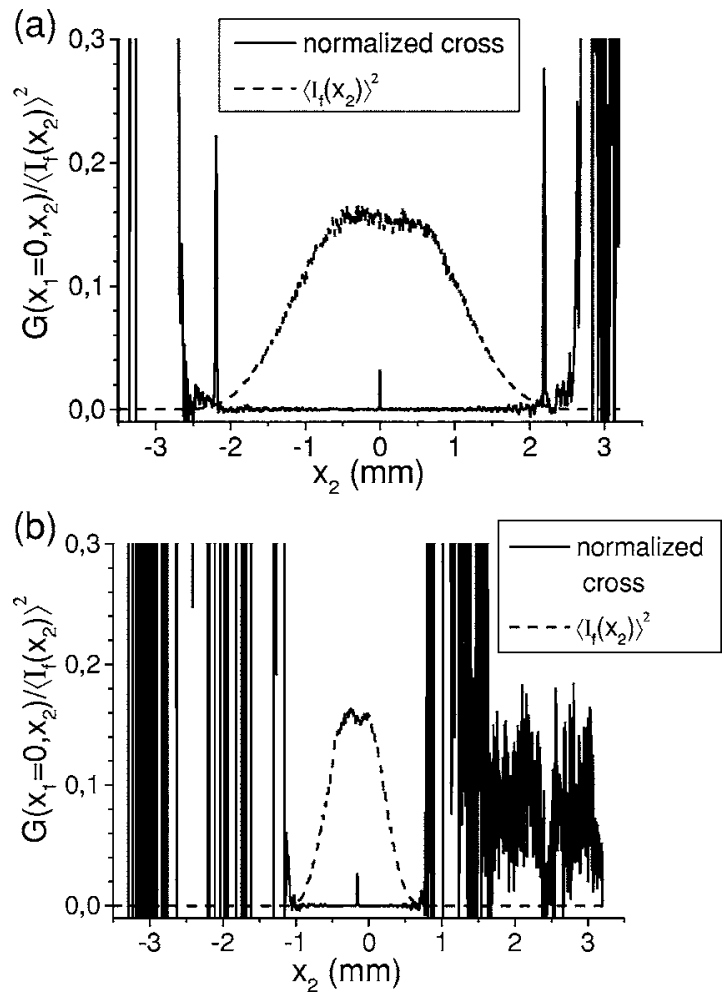

FIG. 11. Cross-correlation function normalized to the square of the mean intensity in the reference arm. (a) $z=115 \mathrm{~mm}$, $\Delta x_{\mathrm{n}}=14 \mu \mathrm{m}$ : the \pm 1 peaks can still be reconstructed. (b) $z=300 \mathrm{~mm}, \Delta x_{\mathrm{n}}=33 \mu \mathrm{m}$ : the diffraction peaks disappear in the noise. Thirty thousand averages are performed for both frames.

the cross correlation is normalized, as shown in Fig. 11 (see also Fig. 3), and we notice that in the case of $\Delta x_{\mathrm{n}}=14 \mu \mathrm{m}$ the \pm 1 peaks can be almost reconstructed, while for $\Delta x_{\mathrm{n}}=33 \mu \mathrm{m}$ they disappear in the noise.

In other words, by increasing the coherence, the signal-tonoise ratio for the reconstruction of the higher order peaks decreases, and the information about the diffraction pattern becomes less and less accessible. To make this argument more formal, we remind that the signal-to-noise ratio is proportional to the visibility defined by Eq. (6), as derived in Ref. [24]. By using Eq. (8), we can readily conclude that for $G$ small the visibility of the $n$ th-order peak, located at $\quad \mathbf{x}_{2}=\overline{\mathbf{x}}_{2}, \quad$ is $\mathcal{V} \approx G\left(\mathbf{x}_{1}, \overline{\mathbf{x}}_{2}\right) /\left[\left\langle I_{1}\left(\mathbf{x}_{1}\right)\right\rangle\left\langle I_{2}\left(\overline{\mathbf{x}}_{2}\right)\right\rangle\right] \approx \eta_{n}\left\langle I_{2}\left(\overline{\mathbf{x}}_{2}\right)\right\rangle /$ $\left\langle I_{1}\left(\mathbf{x}_{1}\right)\right\rangle$. Since the point $\mathbf{x}_{1}$ is fixed at $P_{2}$ where the test intensity is nonzero, the visibility, and hence the signal-tonoise ratio, is proportional to the intensity in the reference arm.

Conversely, by increasing the near-field coherence, the diffraction pattern gradually appears in the autocorrelation function of the test arm, displayed by frames (c) of Figs. 9 and 10. In these plots the diffraction peak values measured via the autocorrelation (triangles) are compared to the values measured by coherent laser illumination (circles). For $\Delta x_{\mathrm{n}}$ $=33 \mu \mathrm{m}$ the partial coherence of the light is already enough to permit an almost perfect pattern reconstruction in the autocorrelation function.

The results presented in this section evidence a clear complementarity between the ghost diffraction scheme and the HBT scheme, which will be further discussed in the next section.

A final remark is the following: had we used the spatial average technique, some information on the diffraction pattern would have been preserved in the correlation when increasing the spatial coherence. In this technique, in fact, the pixel position $\mathbf{x}_{1}$ in the test arm is scanned together with $\mathbf{x}_{2}$; in this way, if some information is present in the test arm intensity distribution, this is retrieved from the correlation. By increasing the spatial coherence, the diffraction pattern becomes visible in the intensity profile of the test arm as shown by Figs. 9(a) and 10(a), and becomes also visible in the correlation as a function of $\mathbf{x}_{1}$. But, obviously, as the diffraction pattern appears in the test arm, is not possible any more to speak about "ghost diffraction."

\section{CONCLUSION}

We have shown that coherent imaging with incoherent classical thermal light is able to produce the interference pattern of a pure phase object. This provides the ultimate demonstration that entanglement is not needed to do coherent imaging with incoherent light, not even in the case of a pure phase object. As our group has pointed out in previous publications [13,17-20,23-25], the only evident advantage of using entangled light might be that of obtaining a better visibility.

A remarkable aspect of the present experiment is the degree of incoherence of the pseudo-thermal speckle beams used. In order to render the beams incoherent with respect to the object (a standard transmission grating beam splitter with 80 grooves per $\mathrm{mm}$ ), we had to create speckles which in the object plane had a size of $2.0 \mu \mathrm{m}$. This was made possible by exploiting the so-called near-field scattering $[29,30]$, in which the speckles are created so close to the source that their size is governed solely by the roughness of the scattering medium.

In such conditions of spatial incoherence, we have shown that no information on the phase object is present in the light outcoming from the object: neither the far-field intensity distribution of the test arm nor its autocorrelation function (HBT scheme) reveal the diffraction pattern. This information is instead present in the cross correlation between the test arm and a reference arm that never passed through the object (ghost diffraction). Our results indeed evidence that, when trying to extract information on a pure phase object, there exists a clear complementarity between the ghost diffraction scheme and the HBT scheme. In the HBT scheme the presence of a certain degree of spatial coherence is the essential ingredient that permits us to extract some phase information, and the information becomes more correct as the coherence increases. Conversely, the ghost diffraction scheme works as a coherent imaging scheme only thanks to the spatial incoherence of the light, and the more the light is incoherent, the better the information is reconstructed. These results contradict what was indicated in the introduction of Ref. [9], where the possibility of doing coherent imaging in a ghost imaging scheme employing split thermal light was ascribed to the presence of spatial coherence. 


\section{ACKNOWLEDGMENTS}

While finalizing this manuscript we were informed of an other experimental observation of a phase object [33] by using split thermal light. This work was carried out in the framework of the FET project QUANTIM of the EU, of the PRIN project of MIUR "Theoretical study of novel devices based on quantum entanglement," and of the INTAS project "Non-classical light in quantum imaging and continuous variable quantum channels." M.B. acknowledges financial support from the Carlsberg Foundation as well as The Danish Natural Science Research Council (FNU, Grant No. 21-04-0506).
[1] D. N. Klyshko, Zh. Eksp. Teor. Fiz. 94, 82 (1988), [Sov. Phys. JETP 67, 1131 (1988)].

[2] A. V. Belinskii and D. N. Klyshko, Zh. Eksp. Teor. Fiz. 105, 487 (1994), [JETP 78, 259 (1994)].

[3] D. V. Strekalov, A. V. Sergienko, D. N. Klyshko, and Y. H. Shih, Phys. Rev. Lett. 74, 3600 (1995).

[4] T. B. Pittman, Y. H. Shih, D. V. Strekalov, and A. V. Sergienko, Phys. Rev. A 52, R3429 (1995).

[5] P. H. S. Ribeiro, S. Padua, J. C. Machado da Silva, and G. A. Barbosa, Phys. Rev. A 49, 4176 (1994).

[6] P. H. Souto Ribeiro, S. Padua, and C. H. Monken, Phys. Rev. A 60, 5074 (1999).

[7] B. E. A. Saleh, A. F. Abouraddy, A. V. Sergienko, and M. C. Teich, Phys. Rev. A 62, 043816 (2000).

[8] A. F. Abouraddy, B. E. A. Saleh, A. V. Sergienko, and M. C. Teich, Phys. Rev. Lett. 87, 123602 (2001); J. Opt. Soc. Am. B 19, 1174 (2002).

[9] A. F. Abouraddy, P. R. Stone, A. V. Sergienko, B. E. A. Saleh, and M. C. Teich, Phys. Rev. Lett. 93, 213903 (2004).

[10] R. S. Bennink, S. J. Bentley, and R. W. Boyd, Phys. Rev. Lett. 89, 113601 (2002).

[11] R. S. Bennink, S. J. Bentley, R. W. Boyd, and J. C. Howell, Phys. Rev. Lett. 92, 033601 (2004).

[12] A. Gatti, E. Brambilla, and L. A. Lugiato, Phys. Rev. Lett. 90, 133603 (2003).

[13] A. Gatti, E. Brambilla, M. Bache, and L. A. Lugiato, Phys. Rev. Lett. 93, 093602 (2004); Phys. Rev. A 70, 013802 (2004).

[14] A. Gatti, E. Brambilla, and L. A. Lugiato, in Quantum Communications and Quantum Imaging, Vol. 5161 of Proc. of SPIE, edited by R. E. Meyers and Y. Shih (SPIE, Bellingham, WA, 2004), p. 192.

[15] M. Bache, E. Brambilla, A. Gatti, and L. A. Lugiato, Phys. Rev. A 70, 023823 (2004).

[16] M. Bache, E. Brambilla, A. Gatti, and L. A. Lugiato, Opt. Express 12, 6067 (2004).

[17] E. Brambilla, A. Gatti, M. Bache, and L. Lugiato, Fortschr. Phys. 52, 1080 (2004).

[18] L. A. Lugiato, A. Gatti, E. Brambilla, and M. Bache, in Fluctuations and Noise in Photonics and Quantum Optics II, Vol. 5468 of Proc. of SPIE, edited by P. Heszler, D. Abbott, J. R. Gea-Banacloche, and P. R. Hemmer (SPIE, Bellingham, WA, 2004), p. 262.

[19] A. Gatti, E. Brambilla, M. Bache, and L. A. Lugiato, Laser Phys. 15, 176 (2005).

[20] M. Bache, L. Lugiato, A. Gatti, and E. Brambilla, in Proceed- ings for II International Conference "Frontiers of Nonlinear Physics,” edited by A. Livtak (IAP RAS, Nizhny Novgorod, Russia, 2005), p. 80.

[21] R. Hanbury-Brown and R. Q. Twiss, Nature (London) 177, 27 (1956).

[22] J. Cheng and S. Han, Phys. Rev. Lett. 92, 093903 (2004).

[23] F. Ferri, D. Magatti, A. Gatti, M. Bache, E. Brambilla, and L. A. Lugiato, Phys. Rev. Lett. 94, 183602 (2005).

[24] A. Gatti, M. Bache, D. Magatti, E. Brambilla, F. Ferri, and L. A. Lugiato, J. Mod. Opt. 53, 739 (2006).

[25] M. Bache, A. Gatti, E. Brambilla, D. Magatti, F. Ferri, and L. Lugiato, quant-ph/0504081.

[26] A. Valencia, G. Scarcelli, M. D’Angelo, and Y. Shih, Phys. Rev. Lett. 94, 063601 (2005).

[27] K. Wang and D.-Z. Cao, Phys. Rev. A 70, 041801(R) (2004).

[28] Y. Cai and S.-Y. Zhu, Opt. Lett. 29, 2716 (2004).

[29] M. Giglio, M. Carpineti, and A. Vailati, Phys. Rev. Lett. 85, 1416 (2000).

[30] M. Giglio, D. Brogiol, M. A. C. Potenza, and A. Vailati, Phys. Chem. Chem. Phys. 6, 1547 (2004).

[31] J. W. Goodman, Introduction to Fourier Optics, 2nd ed. (McGraw-Hill, New York, 1996).

[32] J. W. Goodman, in Laser Speckle and Related Phenomena, Vol. 9 of Topics in Applied Physics, edited by D. Dainty (Springer, Berlin, 1975), p. 9.

[33] R. Borghi, F. Gori, and M. Santarsiero, private communication (2005).

[34] It should be remarked that the HBT correlation vanishes in the limit of full coherence, where the second-order correlation $\left\langle I_{1} I_{2}\right\rangle$ factorizes so that $G_{\mathrm{HBT}}=0$. However, this presumes both spatial and temporal coherence. What we intend here, instead, is simply that we have large speckles on the object, implying thus spatial coherence; the light is however temporally incoherent.

[35] In fact, the observed diffraction pattern for a given speckle size can be understood as the Fourier transform of not the infinitely extended grating, but instead the part of the grating that the speckle is able to "see" due to its finite coherence. Thus, e.g., $\left\langle I_{1}\right\rangle$ obtained for $\Delta x_{\mathrm{n}}=48 \mu \mathrm{m}$ corresponds (roughly) to the diffraction pattern of a transmission grating with four periods.

[36] Note that $z=18 \mathrm{~mm}$ is only the geometrical distance, and the presence of the half-inch glass cube BS should be taken into account. In particular, when calculating the size of FFS or of $D^{*}$, the effective distance $z_{\text {eff }}$ that one should use is shorter than $z$, because of the smaller divergence of light in glass than in vacuum. In our case we estimate $z_{\text {eff }} \approx 14 \mathrm{~mm}$. 